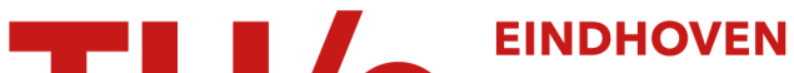 \\ UNIVERSITY OF \\ TECHNOLOGY
}

\section{Crystal shapes and crystallization in continuum modeling}

Citation for published version (APA):

Hütter, M., Rutledge, G. C., \& Armstrong, R. C. (2004). Crystal shapes and crystallization in continuum modeling. Physics of Fluids, 17(1), 014107-1/13. https://doi.org/10.1063/1.1830512

DOI:

10.1063/1.1830512

Document status and date:

Published: 01/01/2004

Document Version:

Publisher's PDF, also known as Version of Record (includes final page, issue and volume numbers)

Please check the document version of this publication:

- A submitted manuscript is the version of the article upon submission and before peer-review. There can be important differences between the submitted version and the official published version of record. People interested in the research are advised to contact the author for the final version of the publication, or visit the $\mathrm{DOI}$ to the publisher's website.

- The final author version and the galley proof are versions of the publication after peer review.

- The final published version features the final layout of the paper including the volume, issue and page numbers.

Link to publication

\section{General rights}

Copyright and moral rights for the publications made accessible in the public portal are retained by the authors and/or other copyright owners and it is a condition of accessing publications that users recognise and abide by the legal requirements associated with these rights.

- Users may download and print one copy of any publication from the public portal for the purpose of private study or research.

- You may not further distribute the material or use it for any profit-making activity or commercial gain

- You may freely distribute the URL identifying the publication in the public portal.

If the publication is distributed under the terms of Article $25 \mathrm{fa}$ of the Dutch Copyright Act, indicated by the "Taverne" license above, please follow below link for the End User Agreement:

www.tue.nl/taverne

Take down policy

If you believe that this document breaches copyright please contact us at:

openaccess@tue.nl

providing details and we will investigate your claim. 


\title{
Crystal shapes and crystallization in continuum modeling
}

\author{
Markus Hütter, ${ }^{\text {a) }}$ Gregory C. Rutledge, ${ }^{\text {b) }}$ and Robert C. Armstrong ${ }^{\text {c) }}$ \\ Department of Chemical Engineering, Massachusetts Institute of Technology, Cambridge, \\ Massachusetts 02139
}

(Received 2 June 2004; accepted 8 October 2004; published online 15 December 2004)

\begin{abstract}
A crystallization model appropriate for application in continuum modeling of complex processes is presented. As an extension to the previously developed Schneider equations [W. Schneider, A. Köppel, and J. Berger, "Non-isothermal crystallization of polymers," Int. Polym. Proc. 2, 151 (1988)], the model presented here allows one to account for the growth of crystals of various shapes and to distinguish between one-, two-, and three-dimensional growth, e.g., between rod-like, plate-like, and sphere-like growth. It is explained how a priori knowledge of the shape and growth processes is to be built into the model in a compact form and how experimental data can be used in conjunction with the dynamic model to determine its growth parameters. The model is capable of treating transient processing conditions and permits their straightforward implementation. By using thermodynamic methods, the intimate relation between the crystal shape and the driving forces for phase change is highlighted. All these capabilities and the versatility of the method are made possible by the consistent use of four structural variables to describe the crystal shape and number density, irrespective of the growth dimensionality. (C) 2005 American Institute of Physics.
\end{abstract}

[DOI: $10.1063 / 1.1830512]$

\section{INTRODUCTION}

Realistic material processing conditions are usually complex, and their modeling is a formidable task. Model development requires appropriate methods, among them of paramount importance the choice of variables. In processes that involve liquid-solid phase transitions, the characterization of the degree of crystallinity is a first key element. In the next level of refinement, characterization of the microstructure, e.g., shape and number of crystallites, is required for a more complete understanding of the morphology, and hence of the material properties of the heterogeneous liquid-solid material as it is formed. A next major step concerns the formulation of dynamic equations for the full set of morphological variables. In this regard, nonequilibrium thermodynamics methods can provide helpful guidelines to comply with fundamental principles of thermodynamics in dynamic situations.

These issues are especially important in the processing of polymers, where flow-induced crystallization occurs. ${ }^{1}$ The combination of microstructure and coupling to flow crucially affects the processing and final materials properties. ${ }^{2,3}$ Due to the relatively long structural relaxation times of polymers as compared to low molecular weight liquids, they show memory effects on experimentally accessible time scales, and conventional flow conditions result in significant distortion of their structure. As a consequence of chain orientation and stretch, the polymers crystallize more easily in flow than in quiescent conditions. Depending on the kinematics, polymers crystallize in various shapes, e.g., spherulites, axialites,

\footnotetext{
${ }^{a)}$ Electronic mail: mhuetter@mit.edu

${ }^{\text {b) }}$ Author to whom correspondence should be addressed. Telephone: (617)

253-0171. Fax: (617) 258-8992. Electronic mail: rutledge@mit.edu

${ }^{c}$ Electronic mail: rca@mit.edu
}

and so-called shish kebabs. ${ }^{2,3}$ The nucleation and growth of the latter depend on the deformation rate and time., ${ }^{3,4}$ This example of flow-induced crystallization of polymers illustrates the two necessary requirements of a successful model. First, the model must distinguish different crystallite shapes; and, second, the rate at which they are nucleated and grow should reflect the processing conditions. Substantial progress has been made in the characterization of the crystal morphology for the crystallization of polymers, both in quiescent and flow situations. ${ }^{3,5-7}$ In the form of four coupled differential equations, the models used in Refs. 3 and 5-7 capture the volume, surface area, average radius, and number of crystallites for a polydisperse distribution of spheres and shish kebabs, respectively.

In this paper, the morphological analysis proposed for polymer crystallization ${ }^{3,5-7}$ is extended to model crystals of arbitrary convex shape, where this shape may depend on processing conditions. In macroscopic modeling, the rheology of polymers is conveniently expressed in terms of measures of chain deformation, where the latter is influenced by the flow field and in turn affects the stress in the system. The degree of chain deformation strongly affects the crystallization process. Therefore, the goal is to develop a crystallization model on the macroscopic continuum scale, with the ability to incorporate shape and nucleation rate and growth rate information from both experiments and microscopic simulations, as shown in Fig. 1. To do so, morphological characterization tools from integral geometry are employed. Dynamic equations sensitive to the crystal shape are proposed and examined under transient conditions. Although inspired by polymer crystallization modeling, the method presented here is more general.

The manuscript is organized as follows. After a brief introduction into the integral geometric characterization 


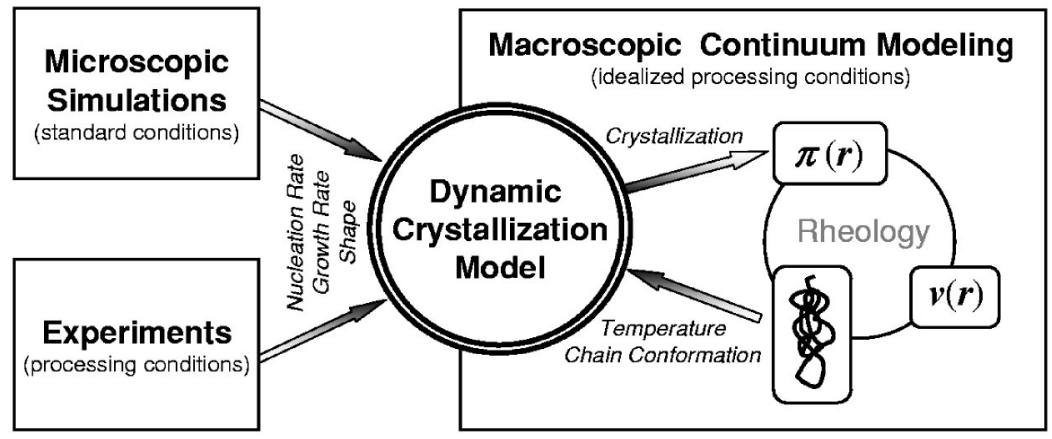

FIG. 1. Dynamic crystallization model embedded in a macroscopic modeling approach with the ability to incorporate data and shape information from experiments and microscopic simulations. methods and dynamic crystallization models in Sec. II, the formulation of a shape sensitive crystallization model is proposed and discussed in detail in Sec. III, including transient conditions. The connection to and verification with experimental data is considered in Sec. V, while Sec. VI discusses the intimate relation between crystal shape and thermodynamic driving forces for crystallization. Conclusions are drawn in Sec. VII.

\section{METHODS}

\section{A. Shape recognition in integral geometry: Minkowski functionals}

Minkowski functionals, also known as intrinsic volumes ("quermassintegrals," curvature integrals), are used in integral geometry to characterize surfaces and shapes. We give here a brief introduction only; for more details the reader is referred to the literature. ${ }^{8-11}$ If $A$ denotes a compact domain in $\mathcal{R}^{d}$ with regular boundary $\partial A \in \mathcal{C}^{2}$, and $d-1$ principal radii of curvature $R_{i}(i=1, \ldots, d-1)$, functionals $W_{\mu}(1 \leqslant \mu \leqslant d)$ can be defined by the following surface integrals:

$$
W_{\mu}(A)=\frac{1}{\mu\left(\begin{array}{l}
d \\
\mu
\end{array}\right)} \int_{\partial A} \mathcal{S}_{\mu-1}\left(\frac{1}{R_{1}}, \ldots, \frac{1}{R_{d-1}}\right) d \mathcal{S},
$$

where $S_{\mu-1}$ is the $(\mu-1)$ th elementary symmetric function and $d \mathcal{S}$ denotes the $(d-1)$-dimensional surface element. In the specific situation of a two-dimensional surface embedded in three-dimensional space we find

$$
\begin{aligned}
& W_{1}=\frac{1}{3} \int_{\partial A} d \mathcal{S}, \quad W_{2}=\frac{1}{3} \int_{\partial A} \frac{1}{2}\left(\frac{1}{R_{1}}+\frac{1}{R_{2}}\right) d \mathcal{S}, \\
& W_{3}=\frac{1}{3} \int_{\partial A} \frac{1}{R_{1} R_{2}} d \mathcal{S} .
\end{aligned}
$$

Note that the integrand in $W_{2}$ is the mean curvature, whereas the integrand in $W_{3}$ is the Gaussian curvature (usually denoted by $H$ and $G$, respectively). Although using the concept of regular surfaces for their definition here, the Minkowski functionals are also well defined for polyhedra with singular edges. ${ }^{9}$ In addition to the above definitions in terms of surface integrals, the zeroth Minkowski functional $W_{0}$ is defined as the volume of the compact domain $A$. Accordingly, the functional $W_{\mu}$ has dimension (length) $)^{d-\mu}(\mu=0,1, \ldots, d)$. To simplify notation for the remainder of the paper, for $d=3$, we also introduce the functionals

$$
\boldsymbol{\Psi}_{\mu}:=\frac{1}{V_{t}} \frac{6}{(3-\mu) !} W_{\mu}, \quad \mu=0,1,2,3,
$$

where $V_{t}$ denotes the total probe volume under consideration. As an example, for a set of $N$ nonoverlapping identical spheres of radius $R$ in $\mathcal{R}^{3}$, the values of the functionals are

$$
\Psi_{\mu, \mathrm{sph}}=\frac{8 \pi}{(3-\mu) !} R^{3-\mu} \frac{N}{V_{t}}, \quad \mu=0,1,2,3 .
$$

In general, the characterization of a domain $A \subset \mathcal{R}^{3}$ as described above results in four numbers, $\boldsymbol{\Psi}_{\mu}(A)$, measuring volume fraction $(\mu=0)$, surface area per unit volume $(\mu=1)$, twice the integrated mean curvature (a characteristic length scale) per unit volume $(\mu=2)$, and $8 \pi$ times the connectivity per unit volume $(\mu=3)$. The latter is defined as the number of disconnected components plus the number of cavities minus the number of tunnels of the domain $A$, i.e., it is a topological measure.

Integral geometric measures in the form of Minkowski functionals find applications in various fields of research. They have been used in complex fluids to study chemical reaction-diffusion patterns, spinodal decomposition kinetics, and the structural phase diagrams of emulsions, ${ }^{12,13}$ as well as to quantify higher order correlations of galaxy cluster distributions in cosmology ${ }^{14,15}$ and the heterogeneity of colloidal particle networks. ${ }^{16}$ The application of these measures also enters into continuum models for crystallization, assuming that the shape of the crystal phase can be described as an assembly of impinging, randomly oriented and randomly positioned, convex crystals. Quiescent as well as flow-induced crystallization of polymers has been modeled, ${ }^{3,5-7}$ and the thermodynamic driving forces for nucleation and growth have been elaborated. ${ }^{17,18}$

\section{B. Crystallization: Integral formulation versus differential formulation}

The theory to determine the overall crystallinity as developed by Kolmogorov, ${ }^{19}$ Johnson and Mehl, ${ }^{20}$ and Avrami $^{21-23}$ is widely used. It relates the volume fraction of the crystalline phase $\phi(t)$ at time $t$ to the nucleation rate per unit volume $\alpha(t)\left(\mathrm{m}^{-3} \mathrm{~s}^{-1}\right)$ through

$$
\phi(t)=1-e^{-\boldsymbol{\Psi}_{0}(t)},
$$




$$
\boldsymbol{\Psi}_{0}(t):=\int_{-\infty}^{t} \alpha\left(t^{\prime}\right) v\left(t^{\prime}, t\right) d t^{\prime}
$$

where $v\left(t^{\prime}, t\right)$ denotes the volume at time $t$ of a single crystallite which began to grow from a nucleus at time $t^{\prime}$. The integral $\boldsymbol{\Psi}_{0}$ given by Eq. (5b) is known as the "extended" volume fraction, which can grow without limit, in contrast to the physical volume fraction $\phi$ where $0 \leqslant \phi \leqslant 1$. The difference between extended and physical volume fractions arises from the impingement of the crystals as they grow. Mathematically, the relation between them, Eq. (5a), is well known from the Boolean grain model for convex grains studied in integral geometry. ${ }^{13}$ For simplicity and clarity, we examine only systems without impingement in the following, i.e., where the individual crystals do not overlap as they grow. In that case, the sum of the single crystal properties equals that property of the union of crystals, e.g., $\boldsymbol{\Psi}_{0}$ from Eq. (5b) is then the physical volume fraction. If one wishes to include impingement in a later stage of the model development, the Boolean grain model in the form Eq. (5a) can be applied readily.

For nonoverlapping convex crystals, the physical volume fraction is given by $\boldsymbol{\Psi}_{0}$ in Eq. (5b). For the special case of spherical crystals that grow at a time-dependent (i.e., process-dependent) radial growth rate $G(t)\left[\mathrm{m} \mathrm{s}^{-1}\right]$, Schneider $\mathrm{et} \mathrm{al.}^{24}$ transformed the integral equation for $\boldsymbol{\Psi}_{0}$ into a set of four coupled differential equations by repeated differentiation of $\boldsymbol{\Psi}_{0}$ with respect to time. By using the notation from the preceding section and since $v\left(t^{\prime}, t\right)$ $=(4 \pi / 3) R^{3}\left(t^{\prime}, t\right)$ for spherical crystals with

$$
R\left(t^{\prime}, t\right)=\int_{t^{\prime}}^{t} G(s) d s,
$$

the Minkowski functionals $\boldsymbol{\Psi}_{\mu}$ defined in the preceding section are given by

$$
\boldsymbol{\Psi}_{\mu}(t)=\frac{8 \pi}{(3-\mu) !} \int_{-\infty}^{t} \alpha\left(t^{\prime}\right) R^{3-\mu}\left(t^{\prime}, t\right) d t^{\prime}, \quad \mu=0,1,2,3 .
$$

Schneiders' rate equations can then be written in the compact form $^{3,5,24}$

$$
\begin{aligned}
& \dot{\boldsymbol{\Psi}}_{\mu}(t)=G(t) \boldsymbol{\Psi}_{\mu+1}(t), \quad \mu=0,1,2, \\
& \dot{\boldsymbol{\Psi}}_{3}(t)=8 \pi \alpha(t) .
\end{aligned}
$$

Here as well as in the remainder of this paper, dotted quantities denote the rate of change exclusively due to the crystallization dynamics. Additional contributions to the dynamic equations of $\boldsymbol{\Psi}_{\mu}$ are not considered here, e.g., the convective terms in general compressible flows which have been discussed elsewhere. ${ }^{17,25-29}$ As required by the integral geometric point of view, the number of dynamic variables in the Schneider equations (8) is four. Note that $\boldsymbol{\Psi}_{\mu}$ captures the $(3-\mu)$ th moment of the crystal size distribution. Therefore, in situations of a polydisperse crystal size distribution, e.g., due to continuous nucleation, the four functionals ( $\mu=0,1,2,3)$ capture different information, again highlighting

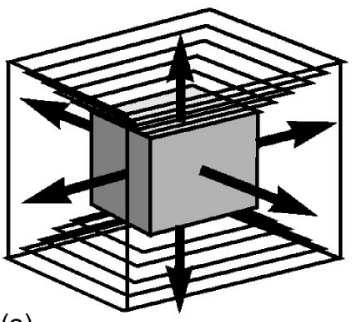

(a)

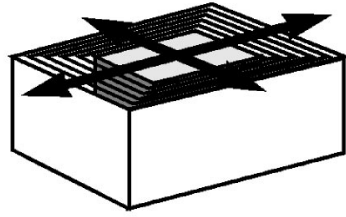

(b)
FIG. 2. Illustration of the dilational growth mode as discussed in the text, exemplified for three-dimensional (a) and two-dimensional growth (b), respectively. The arrows indicate the growth directions.

the need to include them all. Also, the hierarchical differential equations (8) are substantially more useful for implementation in a continuum model and in time-dependent simulations than their integral counterpart (5).

The dynamic equations (8) for the morphological descriptors become physical only when one specifies the material- and process-dependent rates for nucleation $\alpha$ and growth $G$. Apart from fundamentally understanding the driving forces for phase change in terms of differences in chemical potentials, temperatures, and pressures between the solid and liquid phases, ${ }^{17,30-32}$ for practical purposes these rates can be taken from experimental data under standard conditions $^{33,34}$ or from molecular simulations. ${ }^{35-38}$ However, we shall not primarily examine the thermodynamic background of these rates here. Rather, we use a procedure analogous to the one described above to model different particle shapes and different growth dimensionalities.

\section{CRYSTALLIZATION MODEL INCLUDING CRYSTAL SHAPE}

The growth of convex, nonspherical crystals can often be reduced to specifying the shape and a small set of linear growth rates along characteristic directions. For example, once a polymer lamella is recognized to have cylindrical shape, the thickness and radial growth rate are sufficient to characterize its growth. Therefore, the crystallization model presented below is based on the concept of shapes. In the following three Secs. III A, III B, and III C, we discuss crystallization models for growth into three, two, and one dimensions, respectively, with constant crystal shape but timedependent growth rate. Transient situations with variable crystal shape are then studied in Secs. III D and III E, followed by the incorporation of available growth rate data into the model in Sec. III F.

\section{A. Three-dimensional growth}

Under certain conditions, crystals preserve their shape during growth, i.e., the growth process can be described by a dilation in all three directions of the crystals, as shown in Fig. 2(a). Let us assume that all particles, although different in size, are all of the same shape $\mathcal{S}$, the latter being, for example, "cuboid," "ellipsoid," etc. Consider a single crystal and denote its extension along a characteristic axis, which is specific to the shape $\mathcal{S}$, by $2 R$. If the crystal was nucleated at time $t^{\prime}$ and grows in that specific direction at a time- 
TABLE I. Coefficients $\left[c_{0}(\mathcal{S}), c_{1}(\mathcal{S}), c_{2}(\mathcal{S})\right]$ for various shapes in three-dimensional growth $\left(d_{\mathrm{G}}=3\right)$, and $\left(c_{0}\left(\mathcal{S}^{\prime}\right), c_{1}\left(\mathcal{S}^{\prime}\right)\right)$ for various shapes of the parallel surfaces in two-dimensional growth $\left(d_{\mathrm{G}}=2\right)$. The meaning of the parameters $\lambda$ and $\left(\lambda_{1}, \lambda_{2}\right)$, respectively, is illustrated in Fig. 3 for $\left(d_{\mathrm{G}}=3\right)$. The functions $f$ and $g$ are given by $f(x)=x+\ln \left(x+\sqrt{x^{2}-1}\right) / \sqrt{x^{2}-1}$ and $g(x)=x+\arccos (x) / \sqrt{1-x^{2}}$; for $\gamma_{1}\left(\lambda_{1}, \lambda_{2}\right)$ and $\gamma_{2}\left(\lambda_{1}, \lambda_{2}\right)$ the reader is referred to the Appendix. For $d_{\mathrm{G}}=2, \lambda$ denotes the ratio of the two main axes of the ellipse and the rectangle, respectively. $E$ is the complete elliptic integral of the second kind.

\begin{tabular}{|c|c|c|c|c|}
\hline \multirow[b]{2}{*}{$d_{\mathrm{G}}$} & \multirow[b]{2}{*}{ Shape $\mathcal{S}$} & \multicolumn{3}{|c|}{ Coefficients } \\
\hline & & $c_{0}(\mathcal{S})$ & $c_{1}(\mathcal{S})$ & $c_{2}(\mathcal{S})$ \\
\hline & & $4 \pi$ & & \\
\hline 3 & Spheroid (Ref. 12) & $\frac{4 \pi}{3} \lambda$ & $\lambda>1: 2 \pi \lambda g\left(\frac{1}{\lambda}\right)$ & $\lambda>1: 4 \pi f(\lambda)$ \\
\hline & & & $\lambda<1: 2 \pi \lambda f\left(\frac{1}{\lambda}\right)$ & $\lambda<1: 4 \pi g(\lambda)$ \\
\hline 3 & Triaxial ellipsoid & $\frac{4 \pi}{3} \lambda_{1} \lambda_{2}$ & $\gamma_{1}\left(\lambda_{1}, \lambda_{2}\right)$ & $\gamma_{2}\left(\lambda_{1}, \lambda_{2}\right)$ \\
\hline 3 & Double pyramid & $\frac{8}{3} \lambda$ & $8 \sqrt{\lambda^{2}+1}$ & $8 \sqrt{\lambda^{2}+2} \operatorname{arcoss} \frac{1}{\lambda^{2}+1}+8 \arccos \frac{\lambda^{2}-1}{\lambda^{2}+1}$ \\
\hline 2 & Circle & $\pi$ & $2 \pi$ & $\cdots$ \\
\hline 2 & Ellipse & $\pi \lambda$ & $4 E\left(\sqrt{1-\lambda^{2}}\right)$ & $\cdots$ \\
\hline 2 & Rectangle & $\lambda$ & $2(1+\lambda)$ & $\cdots$ \\
\hline
\end{tabular}

dependent rate $G(t)$, then the length in that direction $R\left(t^{\prime}, t\right)$ is given by (6). The Minkowski functionals $\boldsymbol{\Psi}_{\mu}$ for that single particle are given by $c_{\mu}(\mathcal{S}) R^{3-\mu} / V_{\mathrm{t}}(\mu=0,1,2,3)$ with $c_{3}(\mathcal{S}) \equiv 8 \pi$. It is essential to note that the set of coefficients $\mathbf{c}(\mathcal{S}) \equiv\left[c_{0}(\mathcal{S}), c_{1}(\mathcal{S}), c_{2}(\mathcal{S})\right]$ depends on the shape but not on the size of the crystal. One can thus express the functionals for the assembly of continuously nucleated crystals in the form

$$
\boldsymbol{\Psi}_{\mu}(\mathcal{S})=c_{\mu}(\mathcal{S}) \int_{-\infty}^{t} \alpha\left(t^{\prime}\right) R^{3-\mu}\left(t^{\prime}, t\right) d t^{\prime}, \quad \mu=0,1,2,3,
$$

the number density $\boldsymbol{\Psi}_{3}$ being identical to (7) with $\mu=3$. In order to determine the dynamic equations one repeatedly differentiates the volume fraction $\boldsymbol{\Psi}_{0}$. After rearranging terms, one finds

$$
\dot{\boldsymbol{\Psi}}_{\mu}(\mathcal{S})=(3-\mu) \frac{c_{\mu}(\mathcal{S})}{c_{\mu+1}(\mathcal{S})} G(t) \boldsymbol{\Psi}_{\mu+1}(\mathcal{S}), \quad \mu=0,1,2
$$

The evolution equation for the number density $\boldsymbol{\Psi}_{3}$ is always given by (8b), independent of the shape $\mathcal{S}$. Although the crystals grow three dimensionally, only a single growth rate is required in (10) due to the assumption that the shape is a constant of growth. These equations again highlight the hierarchical structure of the model as well as where and how the knowledge of the particle shape is built in, namely, in the coefficients $\mathbf{c}(\mathcal{S})$. For a list of selected shapes these coefficients are given in Table I for illustrative purposes, while the meanings of the length scale $R$ and of the parameters for these shapes are illustrated in Fig. 3. Using the coefficients given in Table I for spherical shape, the growth equations (10) reduce to the Schneider rate equations (8a).

\section{B. Two-dimensional growth}

In this section, we study prism shaped crystals, i.e., crystals having identical and parallel top and bottom bases of shape $\mathcal{S}^{\prime}$ separated by height $h$, which are connected by a

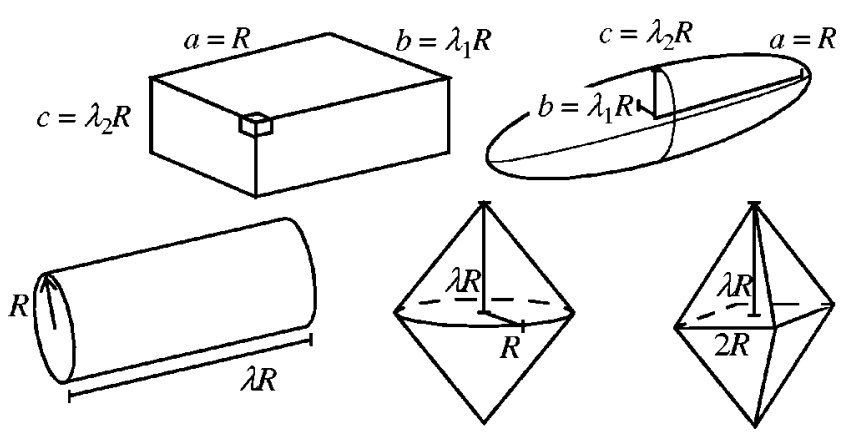

FIG. 3. Illustration of the notation as introduced in the text for five characteristic shapes: rectangular parallelepipedon, triaxial ellipsoid, cylinder, double cone, and double pyramid. 
perpendicular lateral surface. These shapes include cylinders $\left(\mathcal{S}^{\prime}\right.$ : circle) and rectangular parallelepipedons ( $\mathcal{S}^{\prime}$ : rectangle). In contrast to Sec. III A, it is assumed that the thickness of the crystals $h$ does not change due to crystallization and that their growth leads to a dilation of the crystal only in the two remaining planar directions, leaving the two-dimensional shape $\mathcal{S}^{\prime}$ unchanged [see Fig. 2(b)]. Note, however, that the aspect ratios of the three-dimensional shape of the crystallites change upon crystallization, because the thickness $h$ is constant. Let us assume that, in addition to having equal thickness, all crystals also have the same shape $\mathcal{S}^{\prime}$ in the directions perpendicular to the thickness direction, and address the question of suitable functionals for describing an assembly of such particles. In principle, one may choose to use the four morphological variables as defined in (2) and (3), as it has been done in the preceding Sec. III A. However, there are other choices which are more appropriate in view of a crystallization model for two-dimensional growth. The derivation of the hierarchical crystallization model by repeated differentiation (see Sec. III A) suggests that the number of crystallization equations (nucleation and growth) naturally arises as $d_{\mathrm{G}}+1$ for $d_{\mathrm{G}}$-dimensional growth. Hence the number of morphological variables used in the crystallization model is also $d_{\mathrm{G}}+1$. While $d_{\mathrm{G}}+1$ corresponds to the total number of Minkowski functionals for $d_{\mathrm{G}}=3$, this is not the case for $d_{\mathrm{G}}<3$, the consequence of which is discussed in the following.

Let $R$ denote half the extension of a crystal along a characteristic axis specific to the planar shape $\mathcal{S}^{\prime}$ (e.g., radius for a cylinder with circular base shape), and $G(t)$ the timedependent growth rate in that direction. Using the expression (6) for $R\left(t^{\prime}, t\right)$, we define in analogy to (9)

$$
\boldsymbol{\Psi}_{\mu}\left(\mathcal{S}^{\prime}\right)=c_{\mu}\left(\mathcal{S}^{\prime}\right) h(t) \int_{-\infty}^{t} \alpha\left(t^{\prime}\right) R^{2-\mu}\left(t^{\prime}, t\right) d t^{\prime}, \quad \mu=0,1,2,
$$

with $c_{2}\left(\mathcal{S}^{\prime}\right) \equiv 8 \pi$. The coefficients $\left[c_{0}\left(\mathcal{S}^{\prime}\right), c_{1}\left(\mathcal{S}^{\prime}\right)\right]$ are to be chosen such that $\boldsymbol{\Psi}_{0}$ is the volume fraction and $\boldsymbol{\Psi}_{1}$ is the lateral surface area of all particles per unit volume, which is identical to the growth surface. Examples for the coefficients are listed in Table I. Repeated differentiation of the expression (11) for $\mu=0$ results in a set of coupled differential equations, similar to the forms (8b) and (10), namely,

$$
\dot{\boldsymbol{\Psi}}_{\mu}\left(\mathcal{S}^{\prime}\right)=(2-\mu) \frac{c_{\mu}\left(\mathcal{S}^{\prime}\right)}{c_{\mu+1}\left(\mathcal{S}^{\prime}\right)} G(t) \boldsymbol{\Psi}_{\mu+1}\left(\mathcal{S}^{\prime}\right), \quad \mu=0,1,
$$

$$
\dot{\boldsymbol{\Psi}}_{2}\left(\mathcal{S}^{\prime}\right)=8 \pi \alpha(t) h(t)
$$

involving three time-dependent functionals only, $\boldsymbol{\Psi}_{\mu}$ $(\mu=0,1,2)$. However, the morphological description needs to be completed by an additional variable according to integral geometry. We choose the thickness $h(t)$ to be the fourth variable. Although the thickness does not change due to the crystallization dynamics,

$$
\dot{h}=0,
$$

it may still change in time due to other effects, such as applied stress. Therefore, including the thickness variable $h$ in the set of dynamic variables puts one in a position to incorporate such behavior. Furthermore, including the variable $h$ allows one, first, to express the number density of crystals as $\boldsymbol{\Psi}_{2}(t) / h(t)$, which in turn satisfies the common nucleation equation (8b). Second, the total crystal surface area $\boldsymbol{\Psi}_{1}^{\text {total }}$ can be expressed in terms of the growth surface area $\boldsymbol{\Psi}_{1}$, namely,

$$
\boldsymbol{\Psi}_{1}^{\text {total }}=\boldsymbol{\Psi}_{1}+2 \frac{\boldsymbol{\Psi}_{0}}{h}
$$

where the second term accounts for the base surface areas. On one hand, the total crystal surface area can be measured experimentally under certain conditions as discussed below. On the other hand, calculating the free energy of a crystal with different surface tensions for the lateral and base surfaces (e.g., in the case of a lamellar crystal) requires separate knowledge of the lateral and base surface areas, $\boldsymbol{\Psi}_{1}$ and $2 \boldsymbol{\Psi}_{0} / h$, respectively. This clearly highlights the benefit of the choice and number of variables in this section, which allows one to calculate all three surface areas.

\section{One-dimensional growth}

Finally, the situation of growth in only one direction is considered. The extension of a crystal along its growth direction is denoted by $R$ and its time-dependent growth rate by $G$, which includes the growth of both ends. Perpendicular to the growth direction, the base surfaces (and hence the cross section) can be of arbitrary shape $\mathcal{S}^{\prime \prime}$, which are taken identical for all crystals. Since crystals have regular structure, let us assume that for a given cross sectional shape $\mathcal{S}^{\prime \prime}$ one can assign two main axes in that cross sectional plane. Given the extensions of the crystal in these two directions, namely, $h_{1}$ and $h_{2}$, the area of the cross section is of the form $c_{0}\left(\mathcal{S}^{\prime \prime}\right) h_{1} h_{2}$. The discussion of two-dimensional growth has already shown that the definition of the morphological functionals differs from the common integral geometric definitions (2) in order to describe the physics more conveniently. In particular, a crystallization model with one-dimensional growth will require only two dynamic variables, which we define as

$$
\begin{gathered}
\boldsymbol{\Psi}_{\mu}\left(\mathcal{S}^{\prime \prime}\right)=c_{\mu}\left(\mathcal{S}^{\prime \prime}\right) h_{1}(t) h_{2}(t) \int_{-\infty}^{t} \alpha\left(t^{\prime}\right) R^{1-\mu}\left(t^{\prime}, t\right) d t^{\prime}, \\
\mu=0,1,
\end{gathered}
$$

with $R\left(t^{\prime}, t\right)$ as given in (6), and $c_{1} \equiv 8 \pi$. As in the previous Secs. III A and III B the volume fraction is given by $\boldsymbol{\Psi}_{0}$, whereas $\boldsymbol{\Psi}_{1}$ here physically represents $4 \pi / c_{0}$ times the total growth surface area of all crystallites. Repeated differentiation of $\boldsymbol{\Psi}_{0}$ results in the crystallization model with onedimensional growth,

$$
\dot{\boldsymbol{\Psi}}_{0}\left(\mathcal{S}^{\prime \prime}\right)=\frac{c_{0}\left(\mathcal{S}^{\prime \prime}\right)}{8 \pi} G(t) \boldsymbol{\Psi}_{1}\left(\mathcal{S}^{\prime \prime}\right),
$$




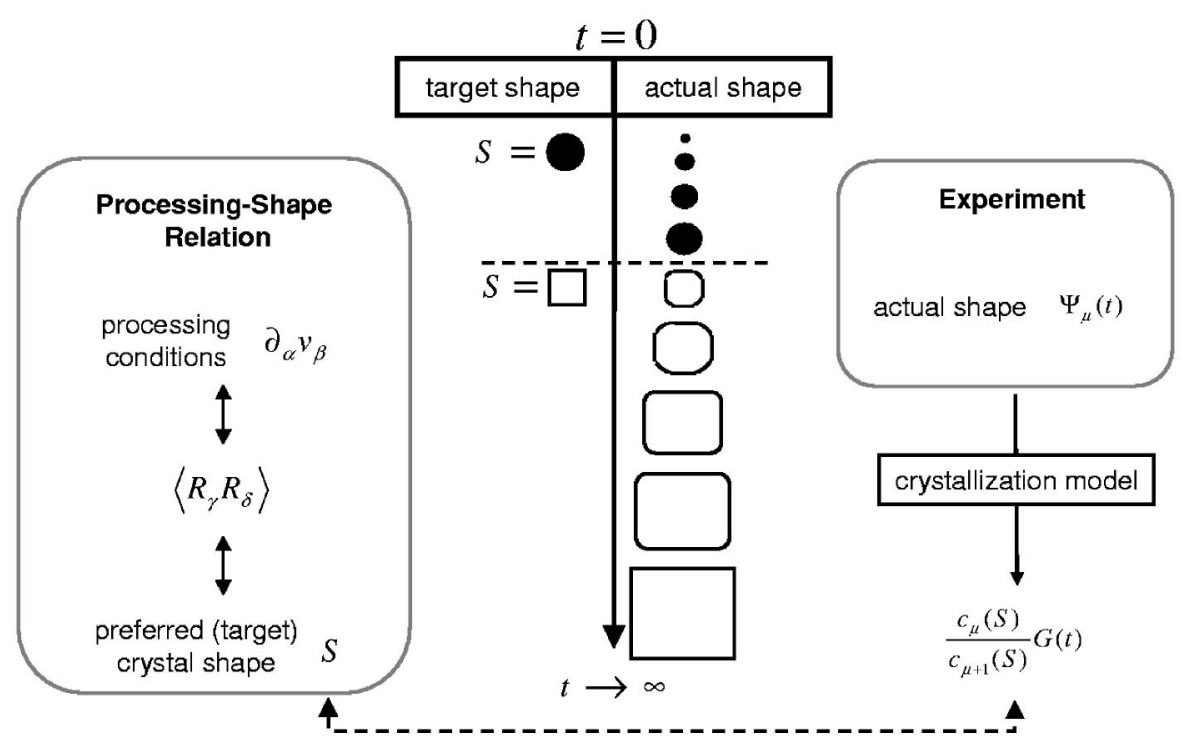

FIG. 4. Illustration of the difference between the target shape and the actual shape as occurring in transient situations. Processing conditions influence the target shape, while experimental techniques measure the actual shape. However, actual and target shape are connected through the crystallization model.

$$
\dot{\boldsymbol{\Psi}}_{1}\left(\mathcal{S}^{\prime \prime}\right)=8 \pi \alpha(t) h_{1}(t) h_{2}(t),
$$

involving two time-dependent functionals only, $\boldsymbol{\Psi}_{\mu}(\mu=0,1)$. While the latter continuously change due to crystallization, the neutral extensions of the crystals $\left(h_{1}, h_{2}\right)$ are unchanged by crystallization,

$$
\dot{h}_{1}=\dot{h}_{2}=0 .
$$

However, $h_{1}$ and $h_{2}$ still need to be included in the set of dynamic variables of the full model in order to control the two planar nongrowth dimensions of the crystal. The axes along which $h_{1}$ and $h_{2}$ are measured are best chosen along the symmetry axes of the crystal structure, for which the anisotropic crystal properties are captured most easily. With the aid of these two additional variables, the number density of crystals can be captured by $\boldsymbol{\Psi}_{1} / h_{1} h_{2}$ which satisfies the common nucleation equation (8b). Furthermore, the total particle surface area $\boldsymbol{\Psi}_{1}^{\text {total }}$ can be written as

$$
\boldsymbol{\Psi}_{1}^{\text {total }}=\frac{c_{0}\left(\mathcal{S}^{\prime \prime}\right)}{4 \pi} \boldsymbol{\Psi}_{1}+\partial c_{0}\left(\mathcal{S}^{\prime \prime}, h_{1}, h_{2}\right) \frac{\boldsymbol{\Psi}_{0}}{c_{0}\left(\mathcal{S}^{\prime \prime}\right) h_{1} h_{2}} .
$$

The first term accounts for the two growth faces and the second term is the lateral surface area with $\partial c_{0}\left(\mathcal{S}^{\prime \prime}, h_{1}, h_{2}\right)$ being the circumference of the cross section. Note that, in contrast to the case of two-dimensional growth (14), this expression (18) explicitly requires $c_{0}$ and $\partial c_{0}$, in addition to all dynamic variables $\left(\boldsymbol{\Psi}_{0}, \boldsymbol{\Psi}_{1}, h_{1}, h_{2}\right)$.

\section{Transient behavior}

The morphological variables given by Eqs. (9), (11), and (15) are solutions to the crystallization equations discussed in the previous Secs. III A-III C assuming that the shape does not change in three-, two-, and one-dimensional growth, respectively, even if the growth rate $G(t)$ varies with time. For many materials, however, the preferred crystal shape depends on processing conditions and thus does change in time. In the picture painted here, such effects are captured by letting the coefficients $\mathbf{c}=\left(c_{0}, \ldots, c_{d_{\mathrm{G}^{-}}}\right)$in the dynamic crystallization equations depend on the processing conditions.

A prototype for transient behavior consists of a step change in the shape coefficients $\mathbf{c}$ at $t=0$, while both the dimensionality $d_{\mathrm{G}}$ and the nongrowth directions are identical for $t \leqslant 0$ and $t>0$. (Alterations of growth dimensionality and directions are discussed in the following section.) Assume that for $t \leqslant 0$ the set of coefficients is $\mathbf{c}^{-}$, whereas for $t>0$ it is $\mathbf{c}^{+}$. Obviously, the crystals nucleated at $t>0$ experience the conditions $\mathbf{c}^{+}$only, and thus develop a shape that conforms to these coefficients from the time of their creation. However, it is interesting to ask what shape the crystals nucleated at $t \leqslant 0$ will develop. To answer this, we examine the solution to the dynamic equations for $d_{\mathrm{G}}$-dimensional growth, namely Eqs. (8b), (10), (12), and (16), respectively, neglecting nucleation and with constant coefficients $\mathbf{c}^{+}$for $t$ $>0$. One finds

$$
\begin{aligned}
\boldsymbol{\Psi}_{\mu}(t>0) & =\sum_{\nu=\mu}^{d_{\mathrm{G}}} \frac{\left(d_{\mathrm{G}}-\mu\right) !}{(\nu-\mu) !\left(d_{\mathrm{G}}-\nu\right) !} R(0, t)^{\nu-\mu} \frac{c_{\mu}^{+}}{c_{\nu}^{+}} \boldsymbol{\Psi}_{\nu}^{-}(0), \\
\mu & =0, \ldots, d_{\mathrm{G}},
\end{aligned}
$$

where the superscript "-" in $\boldsymbol{\Psi}_{\nu}^{-}(0)$ is a reminder that these values are influenced by the coefficients $\mathbf{c}^{-}$only, i.e., by the conditions prior to $t=0$, in contrast to the conditions $\mathbf{c}^{+}$ which apply for $t>0$. The long-time behavior of (19) is dominated by the term $\nu=d_{\mathrm{G}}$, which is proportional to $\boldsymbol{\Psi}_{d_{\mathrm{G}}^{-}}^{-}(0)$, i.e., to the number density of crystallites $\boldsymbol{\Psi}_{3}^{-}(0)$ according to Secs. III A-III C. Thus, Eq. (19) shows that the limiting behavior of all $d_{\mathrm{G}}+1$ variables, $\lim _{t \rightarrow \infty} \boldsymbol{\Psi}_{\mu}(t)$, depends only on the number density of particles at $t=0$. In other words, the crystals lose all memory of their shape in the growth directions prior to $t=0$ due to overgrowth after a sufficiently long time, as illustrated in Fig. 4. Furthermore, the degree to which the crystal shape conforms to the new conditions $\mathbf{c}^{+}$after a finite time $t$ can be estimated from Eq. (19) by comparing the various terms with different powers of $t$. 


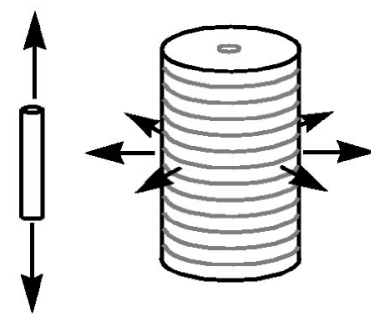

(a)

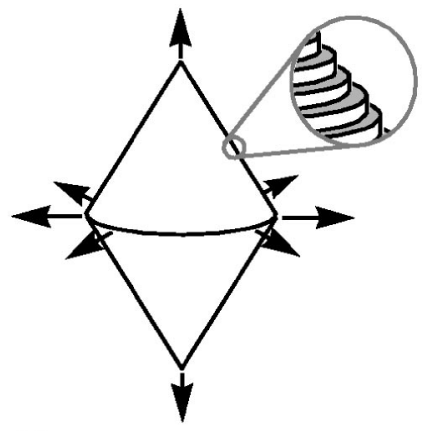

(b)
FIG. 5. Illustration of shish kebab crystallization. For separate or simultaneous shish and kebab growth, the crystals form cylinders (a) or doublecones (b), respectively. The magnified region shows the kebab growth surfaces in white, the integral of which is different from the total surface area of the cone.

In situations of continuously changing coefficients $\mathbf{c}(t)$, the crystal shape always develops toward a shape specified by the current $\mathbf{c}(t)$, but always lags behind in attaining the current shape by virtue of the finite time scale introduced by the growth rate $G$, as demonstrated above. Providing such means to estimate the degree of the lag effect should be useful for the interpretation of experimental data.

\section{E. Alteration of growth directions}

We examine here systems that experience a step change in growth dimensionality and growth directions at $t=0$, but with constant shape coefficients $\mathbf{c}$ before and after that. Such a change is reflected in the initial conditions at $t=0$ for the subsequent growth process. We illustrate this with an example.

In flow-induced crystallization of polymers, the formation of the shish kebabs can, under certain conditions, be decomposed into the flow-induced nucleation and onedimensional growth of shishes $(t<0)$, and the subsequent $(t>0)$ outward growth of the kebabs in two dimensions perpendicular to the initial shish growth direction ${ }^{3,39}$ [see also Fig. 5(a)]. It is assumed here that the flow-induced nucleation and shish length growth is stopped at $t=0$, for example, due to the cessation of flow. For the one-dimensional shish growth of cylindrical shape with radius $r$ at $t<0$, the appropriate variables are $\left(\boldsymbol{\Psi}_{0}^{\mathrm{S}}, \boldsymbol{\Psi}_{1}^{\mathrm{S}}, h_{1}^{\mathrm{S}}, h_{2}^{\mathrm{S}}\right)$ given by Eq. (15) with $c_{0}=\pi$ and $h_{1}^{\mathrm{S}}=h_{2}^{\mathrm{S}}=r$. These variables represent the volume fraction $\left(\boldsymbol{\Psi}_{0}^{\mathrm{S}}\right)$, four times the total growth surface area of all crystallites per unit volume $\left(\boldsymbol{\Psi}_{1}^{\mathrm{S}}\right)$, and the cylinder radius $\left(h_{1}^{\mathrm{S}}, h_{2}^{\mathrm{S}}\right)$. For the two-dimensional kebab growth for $t$ $>0$, the appropriate variables are $\left(\boldsymbol{\Psi}_{0}^{\mathrm{K}}, \boldsymbol{\Psi}_{1}^{\mathrm{K}}, \boldsymbol{\Psi}_{2}^{\mathrm{K}}, h^{\mathrm{K}}\right)$ given by Eq. (11) for a cylinder with $c_{0}=\pi$ and $c_{1}=2 \pi$ (see Table I). These variables stand for the volume fraction $(\mu=0)$, the growth surface area per unit volume $(\mu=1), 8 \pi h^{\mathrm{K}}$ times the number density of crystallites $(\mu=2)$, and the shish length $h^{\mathrm{K}}$. Since the descriptions for shish growth and kebab growth must represent the same structure at $t=0$, one finds

$$
\Psi_{0}^{\mathrm{K}}(0)=\boldsymbol{\Psi}_{0}^{\mathrm{S}}(0)
$$

$$
\begin{aligned}
& \boldsymbol{\Psi}_{1}^{\mathrm{K}}(0)=\frac{2}{h_{1}^{\mathrm{S}}} \boldsymbol{\Psi}_{0}^{\mathrm{S}}(0), \\
& \boldsymbol{\Psi}_{2}^{\mathrm{K}}(0)=\frac{8}{h_{1}^{\mathrm{S}^{2}}} \boldsymbol{\Psi}_{0}^{\mathrm{S}}(0), \\
& h^{\mathrm{K}}(0)=8 \frac{\boldsymbol{\Psi}_{0}^{\mathrm{S}}(0)}{\boldsymbol{\Psi}_{1}^{\mathrm{S}}(0)} .
\end{aligned}
$$

These initial conditions, which show the connection between the two different regimes, can now be used to study the kebab growth in terms of the dynamic equations (12). The key result of this shish-kebab growth example is that the crystallization model presented in Secs. III A-III C can accommodate a change in the growth dimensionality and in the growth directions by appropriately matching the variables at that transition. The explicit use of $h_{1}^{\mathrm{S}}$ in Eq. (20) highlights the importance of also including "neutral" variables in the description, instead of the growth variables only.

\section{F. Incorporating knowledge about growth rates}

Often, one has some information about the crystallization process from experiments or molecular simulations, such as linear growth rates and the accompanying shape. The question arises as to how such information is to be incorporated into the crystallization model.

The dynamic crystallization model, Eqs. (8b), (10), (12), and (16), has already been written in a form suitable for considering crystal growth of specific shapes. The latter are represented in the model in terms of the coefficients $\mathbf{c}(\boldsymbol{\lambda})$, where $\boldsymbol{\lambda}$ denotes the set of parameters for that shape family. Examples for these coefficients are given in Table I. In order to discuss the incorporation of available growth rates into the model, we study the example of a rectangular parallelepipedon shown in Fig. 3. If the growth rates in the three directions are denoted by $\left(G_{a}, G_{b}, G_{c}\right)$, then the shape after infinitely long time is a parallelepipedon with side lengths with ratios $\left(1, \lambda_{1}, \lambda_{2}\right)$ (see also Fig. 3) with

$$
\begin{aligned}
& G=G_{a}, \\
& \lambda_{1}=\frac{G_{b}}{G_{a}}, \\
& \lambda_{2}=\frac{G_{c}}{G_{a}},
\end{aligned}
$$

because the coefficients $\mathbf{c}(\boldsymbol{\lambda})$ with values (21b) and (21c) exactly describe the target shape after a long time, as shown in Sec. III D.

\section{ILLUSTRATIVE EXAMPLES}

\section{A. Extended chain single crystals}

The crystallization of polyethylene under high pressure results in single crystals of tapered shape ${ }^{40,41}$ Starting from a folded primary nucleus, the combination of lateral growth and thickness growth through chain-sliding diffusion leads to 
extended chain single crystals. During this growth the thickness grows linearly with time, as does the lateral size (diameter). Therefore, according to the experimental findings the crystal shape is appropriately described by a double cone, for which the ratio $\lambda$ (see Fig. 3) of total height to diameter is constant. In order to model this crystallization process, the coefficients $\mathbf{c}(\lambda)$ corresponding to a double cone (see Table I) have to be used in the three-dimensional growth model (10), where the value for $\lambda$ can be taken from experimental data, ${ }^{40,41}$ e.g., $\lambda \simeq 1 / 10$. The rate $G$ in the dynamic model represents the lateral growth rate.

\section{B. Thickness growth in lamella stacks}

Experimental results indicate that under certain conditions the crystal thickness neither stays constant nor does it grow linearly in time, as described just above. For example, the thickness of slowly cooled melt-crystallized polyethylene and single crystals grows logarithmically with time upon annealing, and also the thickness growth rate of stacked lamella slows down as the lamella thickness is comparable to the spacing between the lamellae. ${ }^{42,43}$ In order to study how such effects can be represented in the crystallization model, we consider the individual crystals to be lamellae, i.e., of cylindrical shape, with ratio $\lambda$ between cylinder height and radius. Since the thickness growth rate is decreasing with time while the radial growth rate is constant, the value of $\lambda$ in the crystallization model has to change during the crystallization process. In particular, for the case of arrested thickness growth in the long-time limit, one has $\lim _{t \rightarrow \infty} \lambda(t)=0$. The situation of arrested thickness growth for long times can be accounted for as follows. First, we denote the ratio of height to radius for the very early stages of crystallization (i.e., for small crystallites) by $\lambda_{0}$. Second, we remember that in the long time limit we wish to have a constant thickness, $h_{\infty}$, which depends for example on the distance between successive lamellae in the stack. The final thickness $h_{\infty}$ can be approximated by the expression $\lambda\langle R\rangle$ with the average cylinder radius $\langle R\rangle$. Since $4 \boldsymbol{\Psi}_{2} /\left(\pi \boldsymbol{\Psi}_{3}\right)=(1+\lambda / \pi)\langle R\rangle$ (see Table I) is a good measure for the average cylinder radius, in particular as $\lambda$ becomes small, one can make the following ansatz:

$$
\lambda\left(\boldsymbol{\Psi}_{2}, \boldsymbol{\Psi}_{3}\right)=\frac{1}{\left(1 / \lambda_{0}\right)+\left(4 \boldsymbol{\Psi}_{2}\right) /\left(h_{\infty} \pi \boldsymbol{\Psi}_{3}\right)} .
$$

For the initial stages, $\boldsymbol{\Psi}_{2} / \boldsymbol{\Psi}_{3}$ is small (i.e., small crystallites) and hence $\lambda \simeq \lambda_{0}$. In contrast, for the late stages $\boldsymbol{\Psi}_{2} / \boldsymbol{\Psi}_{3}$ is large and hence $\lambda$ is small by virtue of Eq. (22), and therefore $\lambda\left(\boldsymbol{\Psi}_{2}, \boldsymbol{\Psi}_{3}\right)\langle R\rangle \simeq \lambda\left(\boldsymbol{\Psi}_{2}, \boldsymbol{\Psi}_{3}\right) 4 \boldsymbol{\Psi}_{2}\left(/ \pi \boldsymbol{\Psi}_{3}\right) \simeq h_{\infty}$, as intended. Solving the three-dimensional growth model (10) with the cylinder shape coefficients listed in Table I and the ansatz (22) indeed results in the average thickness approaching a constant value for long times. However, this limiting thickness of the actual crystals slightly differs from $h_{\infty} \simeq \lambda\langle R\rangle$ due to the time lag effects discussed in Sec. III D, because the quantity $h_{\infty}$ is related to the target shape through $\lambda$. The saturation of thickness growth is also reflected in the Avrami exponent, defined by the derivative $m$ $=\partial(\ln \Psi 0) / \partial(\ln t)$ in our notation, which gradually transi- tions from an initial value of $m=3$ to $m=2$, in the absence of nucleation for simplicity. Therefore, the dimensionality of growth as measured by the Avrami exponent shows a gradual decrease although the crystals grow in (exactly) three dimensions for all times except for $t \rightarrow \infty$. In turn, noninteger Avrami exponents determined from experimental data can result from a growth nonlinear in time for at least one of the growth directions, in addition to a time variation in nucleation rate.

\section{Shish-kebab growth: Revisited}

In Sec. III E the shish-kebab growth has been used to illustrate how a change in dimensionality can be incorporated in the model. In contrast, Eder and Janeschitz-Kriegl ${ }^{3}$ (EJK) use a slightly different idea. In their case, a kebab starts growing outward immediately from every added shish element, i.e., shish and kebab growth occur simultaneously. Their surface area variable represents the sum of all growth surfaces of the disk-shaped kebabs, i.e., it measures the active area for the deposition of the polymer chains onto the kebab growth surfaces. While time-dependent nucleation and growth rates lead to complicated shapes which are correctly described in the EJK model, a double cone-like shape as illustrated in Fig. 5(b) develops under stationary conditions. In the crystallization model discussed in this paper, double cones are modeled with coefficients $\mathbf{c}$ that correspond to this shape, and have a smooth surface in contrast to the stepped surface in the Eder and Janeschitz-Kriegl model, see Fig. 5(b). The double cone representation is exact for stationary conditions, but only approximate in transient situations. In contrast to the growth surface area variable in the EJK model, the shape model used here for double cones correctly captures the total crystal surface, which is the relevant one for measurement with scattering methods, as discussed below.

The specific goals of the modeling effort dictate which of the two models for simultaneous shish and kebab growth is to be used, since each of these models has its advantages and disadvantages. The choice between models that treat shish and kebab growth simultaneously or sequentially is a physical decision which can be made on the following grounds. Close examination of various flow-induced crystallization experiments indicate that the stretch of the chain backbone $\alpha$ is a critical parameter for the formation of the shish-kebab structure. ${ }^{44}$ Using this hypothesis, we denote the value above which shish formation is observed by $\alpha_{\mathrm{S}}$, whereas kebabs can only form if the chain stretch is below a threshold value, $\alpha_{\mathrm{K}}$. If $\alpha_{\mathrm{K}}<\alpha_{\mathrm{S}}$, there is only sequential shish and kebab growth, while for $\alpha_{\mathrm{K}}>\alpha_{\mathrm{S}}$ a range of stretching exists in which simultaneous shish and kebab growth occurs. As illustrated above, the shape model presented here is capable of describing both the simultaneous and the sequential growth mode, which lead to double cone-like and cylindrical shapes, respectively.

Experiments show that the shish structure, although nucleated only in the stretched melt, can grow into undeformed parts of the melt. ${ }^{45}$ In other words, this phenomena occurs at the boundary of a stretched melt, i.e., in a thin layer 
not larger than the length of the shish. Considering the fact that the resolution of the variables in the current crystallization model (i.e., the volume element) is much larger than the crystal size, special care has to be taken when incorporating such boundary effects. Nevertheless, if one wishes to describe such shish growth into the undeformed melt, there are (at least) two possibilities to do so. First, the preferred crystal shape (i.e., "shish") can be seen as not being determined by the local state of the melt, but rather by nonlocal effects, which in turn have to be reflected in the shape parameters $\boldsymbol{\lambda}$ in the model. Another possibility is to assume the idea of self-generated fields proposed by Schultz. ${ }^{46}$ One then needs to think carefully about the local parameter/property of the melt which is supposed to influence the crystal target shape.

\section{Fibril-lamella transition}

Heat treatment of highly oriented polypropylene and poly(ethylene-terephtalate) brings about a transition from a fibrillar to a lamellar structure. ${ }^{47,48}$ In this transition, several oriented partly crystalline fibrillar crystals merge to form a stack of lamellae, where the stacking direction is along the fibrillar direction and the lamellae themselves are oriented perpendicular. For simplification, we assume that the crystal morphology is described by one-dimensional growth of cylinders prior to the transition, and by two-dimensional (lateral) growth of cylinders thereafter. Thus, in terms of variables, this situation corresponds to the case of the sequential shish-kebab transition of Sec. III E. For the fibrillar and lamellar states we use the variables $\left(\boldsymbol{\Psi}_{0}^{\mathrm{F}}, \mathbf{\Psi}_{1}^{\mathrm{F}}, h_{1}^{\mathrm{F}}, h_{2}^{\mathrm{F}}\right)$ and $\left(\boldsymbol{\Psi}_{0}^{\mathrm{L}}, \boldsymbol{\Psi}_{1}^{\mathrm{L}}, \boldsymbol{\Psi}_{2}^{\mathrm{L}}, h^{\mathrm{L}}\right)$, respectively. Here, $h_{1}^{\mathrm{F}}=h_{2}^{\mathrm{F}}$ is the radius of the fibrils, and $h^{\mathrm{L}}$ the thickness of the lamellae. Although the fibrils are only partly crystalline, we choose the variables with superscript "F" to describe the entire fibrils; $\boldsymbol{\Psi}_{0}^{\mathrm{F}}$ thus describes the filling of space with fibrils, rather than a real degree of crystallinity. In order to connect the fibrillar and lamellar descriptions at the transition, additional experimental data are required: the average number of fibrils $\mathcal{N}$ that merge sideways to form a lamella; the average degree of crystallinity $\varphi$ within one (partly crystalline) fibril; and the periodicity of the defect modulation $\Delta$ in the fibrils prior to the transition, which sets the scale for the periodicity of the lamellar stacking. With these quantities and using the very definitions of the structural variables one arrives at these relations at the transition:

$$
\begin{aligned}
& \boldsymbol{\Psi}_{0}^{\mathrm{L}}=\varphi \boldsymbol{\Psi}_{0}^{\mathrm{F}}, \\
& \boldsymbol{\Psi}_{1}^{\mathrm{L}}=\frac{2 \varphi}{\sqrt{\mathcal{N}} h_{1}^{\mathrm{F}}} \boldsymbol{\Psi}_{0}^{\mathrm{F}}, \\
& \boldsymbol{\Psi}_{2}^{\mathrm{L}}=\frac{8 \varphi}{\mathcal{N} h_{1}^{\mathrm{F}^{2}}} \boldsymbol{\Psi}_{0}^{\mathrm{F}}, \\
& h^{\mathrm{L}}=\varphi \Delta,
\end{aligned}
$$

where the additional assumption has been made that the sum of the cross sectional areas of $\mathcal{N}$ fibrils equals the cross sectional area of a lamella. However, this assumption is not essential here and can be altered if one wishes to do so. In conclusion, relations (23) show that the descriptions of the crystallization prior to and after the complicated structural transition can be linked, but only if certain experimental data is available.

\section{EXPERIMENTAL RELEVANCE}

In contrast to incorporating a priori knowledge of the preferred crystal shape and growth rate into the crystallization model, it is also desirable to measure these properties experimentally, even under transient conditions. Specifically, we discuss what kinds of experimental data are needed to extract the shape of the crystals and to study the relation between processing conditions and preferred crystal shape.

\section{A. Scattering and form factor}

The shape of discrete particles leaves significant fingerprints in scattering experiments, namely in the form factor $P(\mathbf{q})$. For details the reader is referred, for example, to an introduction by Pusey. ${ }^{49}$ We consider here the limit of low number density of crystals, i.e., we assume that multiple scattering can be neglected and that the particle positions and orientations are uncorrelated. For systems of identical particles only, the total scattering intensity depends on the scattering vector $\mathbf{q}$ only due to the form factor. Therefore, the following specific properties of the form factor can be exploited directly for evaluating the experimentally accessible scattering intensity. First, the form factor goes to unity for small q. Second, in the Porod limit ${ }^{50-53} P(\mathbf{q})$ is proportional to the particle surface area divided by the square of the particle volume for isotropic systems. Third, the integral of $P(\mathbf{q})$ over all $\mathbf{q}$ is inversely proportional to the particle volume. However, still not all of the required four structural variables can be measured from these three general features of the form factor. In order to complete these results, one may use precalculated form factors for predetermined shapes and compare them with the experimental data, ${ }^{54}$ which then provides the shape parameters $\boldsymbol{\lambda}$ and in turn the coefficients $\mathbf{c}(\boldsymbol{\lambda})$. Form factors have been calculated for various parametrized shapes (see also Ref. 55), e.g., sphere, ${ }^{56}$ ellipsoid of revolution, ${ }^{57}$ triaxial ellipsoids, ${ }^{58}$ rectangular parallelepipedons, ${ }^{59}$ and cylinder. ${ }^{60}$

For an assembly of particles of unknown (but common) shape and polydisperse size, the simultaneous determination of the shape and size distribution is impossible. ${ }^{54}$ Either the size distribution must be measured by other means in order to determine the particle shape from the scattering experiment, or vice versa. The procedure for measuring the processing-shape relation discussed below thus has certain limitations.

\section{B. Measuring the processing-shape relation}

Before proceeding, it is essential to distinguish between the structural variables as measured experimentally on the one hand, and on the other hand the shape $\mathcal{S}$ and parameters $\boldsymbol{\lambda}$ appearing in the dynamic model (8b), (10), (12), and (16), respectively. While the former reveal information about the 
actual shape, the latter indicates the target shape toward which the crystals evolve (see Fig. 4). As discussed in Sec. III D on the transient behavior, the actual structure lags behind the target shape $\mathcal{S}$ due to the finite growth rate $G(t)$. Although the actual shape is directly accessible in experiments, one has to keep in mind that from a modeling perspective it is the target shape that matters. One may examine the relation between processing conditions, chain deformation, and target shape (see Fig. 4), which is closely related to experiments and simulations under stationary conditions, and is thus often the main focus in modeling. In so doing, the complicated overgrowth effects do not enter the picture; and this distinct physical process can be treated separately. Therefore, the measurement of the target shape $\mathcal{S}$ is discussed below in detail.

First, the dimensionality of growth $d_{\mathrm{G}}$ has to be obtained from the experimental data. For constant growth rate, one finds the value for $d_{\mathrm{G}}$ from the time dependence of the volume fraction (similarly to the determination of the Avrami exponent ${ }^{61}$ ), assuming the nucleation rate is either constant or pulse-like. Under transient conditions, the rates ( $\alpha$ and $G$ ) as well as the target shape ( $\mathcal{S}$ and $\lambda$ ) may change in time due to changing processing conditions. In our model as well as in experiments, such effects result in noninteger values for the Avrami exponent, ${ }^{61,62}$ even though the dimensionality of growth $d_{\mathrm{G}}$ remains an integer. Hence the time dependence of the volume fraction cannot be used for the determination of $d_{\mathrm{G}}$ in transient situations. Instead, $d_{\mathrm{G}}$ is given by the number of independent crystal dimensions that grow in time, and these dimensions must be determined independent of volume fraction by the scattering experiment.

Second, corresponding to the specific value for $d_{\mathrm{G}}$ one must consider the dynamic equations (8b), (10), (12), and (16), respectively, for a shape $\mathcal{S}_{j}$ with the corresponding set of parameters $\boldsymbol{\lambda}^{[j]}$. In the event that experiments can provide the values $\boldsymbol{\Psi}_{\mu} \quad\left(\mu=0, \ldots d_{\mathrm{G}}\right)$ and their rate of change by appropriate scattering data, then the values of the ratios

$$
\begin{gathered}
{\left[\frac{\dot{\boldsymbol{\Psi}}_{\mu}}{\boldsymbol{\Psi}_{\mu+1}}\right]_{\exp }=\left[\left(d_{\mathrm{G}}-\mu\right) \frac{c_{\mu}\left(\boldsymbol{\lambda}^{[j]}\right)}{c_{\mu+1}\left(\boldsymbol{\lambda}^{[j]}\right)} G(t)\right]_{\text {theo }},} \\
\mu=0, \ldots, d_{\mathrm{G}}-1
\end{gathered}
$$

are known. If the family of shapes used in Eq. (24) has $d_{\mathrm{G}}$ -1 parameters, solution of the $d_{\mathrm{G}}$ equations (24) gives a unique solution for the growth rate $G$ and the $d_{\mathrm{G}}-1$ parameters. If the number of parameters is less than $d_{\mathrm{G}}-1$, then the system of constraints (24) is overdetermined. If there is no solution, then the shape of the crystals in the experiment is not given by $\mathcal{S}_{j}$; and one knows to solve (24) for another shape $\mathcal{S}_{k}$ with parameters $\lambda^{[k]}$, where a higher number of parameters may be required. An example of the overdetermined case is the one-parameter family of spheroids, which show three-dimensional growth. In summary, it is up to the user to choose between families of shapes with number of parameters $m \leqslant d_{\mathrm{G}}-1$. If $m<d_{\mathrm{G}}-1$, one has limited flexibility as far as parametrization is concerned, but greater confidence concerning the shape in general, since for the wrong choice of shape family, no solution to (24) exists. If $m=d_{\mathrm{G}}$ -1 , one must assume that the shape is correct and then get the corresponding values for the parameters. These then describe the target crystal shape as required for the processingshape relation (see Fig. 4).

Finally, one should note that this entire procedure and the very existence of the conditions (24) go back to including more morphological variables (and hence more details) in the description than just the commonly used volume fraction.

\section{CONSEQUENCES FOR THERMODYNAMIC DRIVING FORCES}

It has been shown in previous work ${ }^{17,63}$ for specific cases, that the shape of the crystallites is connected to the thermodynamic driving force behind the growth rate. This previous analysis is generalized here in view of the shape dependent crystallization model presented above. In essence the dynamic equations of the forms (8b), (10), (12), and (16) are combined with a two-phase model by using nonequilibrium thermodynamic techniques. ${ }^{64-68}$

The full set of variables ( $\mathbf{z})$ of the combined model includes thermodynamic variables for the melt and crystal phases, such as their mass densities $\rho_{\mathrm{m}}$ and $\rho_{\mathrm{c}}$, as well as the Minkowski functionals. For simplicity three-dimensional growth is considered $\left(d_{\mathrm{G}}=3\right)$ in the following: the other cases can be treated similarly. If one assumes that both phases have equal temperature, then the relevant thermodynamic potential is the Helmholtz free energy, $F(\mathbf{z})$. A change in the volume fraction $\boldsymbol{\Psi}_{0}$ due to crystallization is coupled to mass transfer between the phases, i.e., $\dot{\rho}_{\mathrm{c}}=\hat{\rho} \dot{\Psi}_{0}$ and $\dot{\rho}_{\mathrm{m}}$ $=-\hat{\rho} \dot{\Psi}_{0}$, where $\hat{\rho}$ is a mass density. Taking such dependencies into account, it is useful to define the four functions $\boldsymbol{\Lambda}$ through

$$
\left.\dot{F}(\mathbf{z})\right|_{\dot{\Psi}_{\mu}}=-\Lambda_{\mu}(\mathbf{z}) \dot{\Psi}_{\mu}, \quad \mu=0,1,2,3,
$$

where the right side does not include a summation. The subscript on the left side denotes inclusion of changes in $F$ caused by changes in $\boldsymbol{\Psi}_{\mu}$ due to crystallization. While bulk contributions (e.g., the chemical potential difference of the melt and crystal phase) are contained in $\Lambda_{0}$, surface tension effects are represented in the surface area derivative $\Lambda_{1}$. It has been shown that, in order to respect fundamental thermodynamic principles, the crystallization equations for the Minkowski functionals $\boldsymbol{\Psi}=\left(\boldsymbol{\Psi}_{0}, \boldsymbol{\Psi}_{1}, \boldsymbol{\Psi}_{2}, \boldsymbol{\Psi}_{3}\right)$ must take the form $^{17,63}$

$$
\dot{\Psi}=\mathbf{R} \cdot \Lambda,
$$

where $\mathbf{R}$ is a positive semidefinite and symmetric $4 \times 4$ matrix.

Equation (26) describes the same physics represented by (8b) and (10), according to which the ratio $\dot{\boldsymbol{\Psi}}_{\mu} / \dot{\boldsymbol{\Psi}}_{\nu}$ for $0 \leqslant \mu, \nu \leqslant 2$ is independent of the growth rate $G$, i.e., independent of any thermodynamic driving force. In turn, this means by virtue of Eq. (26) that the ratio of the corresponding rows of $\mathbf{R}$ must satisfy the same condition. Exploiting the symmetry property of that matrix and assuming $\Lambda_{\mu} \neq 0(\mu$ $=0,1,2,3)$, one finally arrives at 


$$
\begin{aligned}
\frac{G}{R_{0}}= & \left(3 c_{0} \Lambda_{0}+2\left[\frac{\boldsymbol{\Psi}_{2} / c_{2}}{\boldsymbol{\Psi}_{1} / c_{1}}\right] c_{1} \Lambda_{1}+\left[\frac{\boldsymbol{\Psi}_{3} / c_{3}}{\boldsymbol{\Psi}_{1} / c_{1}}\right] c_{2} \Lambda_{2}\right) \\
& +P\left[\frac{1}{\boldsymbol{\Psi}_{1} / c_{1}}\right] \Lambda_{3}, \\
\frac{8 \pi \alpha}{R_{0}}= & \left(3 c_{0} \Lambda_{0}+2\left[\frac{\boldsymbol{\Psi}_{2} / c_{2}}{\boldsymbol{\Psi}_{1} / c_{1}}\right] c_{1} \Lambda_{1}+\left[\frac{\boldsymbol{\Psi}_{3} / c_{3}}{\boldsymbol{\Psi}_{1} / c_{1}}\right] c_{2} \Lambda_{2}\right) P \\
& +Q\left[\frac{1}{\boldsymbol{\Psi}_{1} / c_{1}}\right] \Lambda_{3} .
\end{aligned}
$$

Here, $R_{0}(\mathbf{z}) \geqslant 0$ is a general kinetic coefficient, and the functions $P(\mathbf{z})$ and $Q(\mathbf{z})$ are constrained to $Q \geqslant P^{2}$ due to the positivity requirement of the matrix $\mathbf{R}$. Although the terms in brackets in Eq. (27) do not depend on the crystal shape but only on the crystal size, the growth rate and nucleation rate are still shape-dependent. The crucial conclusion is that the shape coefficients $\left(c_{0}, c_{1}, c_{2}\right)$ enter the dynamic equations for the morphological variables not only explicitly, as suggested in (10), but that they also enter into the growth rate $G$ and the nucleation rate $\alpha$ implicitly, as shown in (27). Since this result is a direct consequence of the positivity and symmetry of the matrix $\mathbf{R}$, the relations (27) highlight the intimate coupling of structure and thermodynamics. Also note that whereas $c_{0} \Lambda_{0}$ is a size independent bulk term, the terms proportional to $\Lambda_{1}$ (i.e., surface tension) are size dependent due to the average inverse length scale $\boldsymbol{\Psi}_{2} / \boldsymbol{\Psi}_{1}$. This shows how the size dependences of the growth rate and nucleation rate due to surface tension are captured appropriately on this level of description when using the variables $\boldsymbol{\Psi}_{\mu}(\mu=0,1,2$, $3)$. It also emphasizes again the necessity to include all four functionals in the description in order to describe such effects properly.

The nucleation rate (27b) is proportional to the growth rate (27a) if $Q=P^{2}$, which corresponds to the isokinetic assumption of the Nakamura equation. ${ }^{69}$ Correspondingly, one can show that the rank of the matrix $\mathbf{R}$, which represents the number of physically distinct processes, drops from two for $Q \neq P^{2}$ to one for $Q=P^{2}$. Only in the former case can one speak of a true physical distinction between nucleation and growth.

\section{CONCLUSIONS}

A crystallization model capable of recognizing shape has been introduced in a general manner in Sec. III. It is capable of capturing one-, two-, and three-dimensional growth and of connecting these different regimes, as shown with the shishkebab example. A priori knowledge of the crystal shape under stationary conditions can be incorporated into the dynamic model in a compact form.

Transient crystallization conditions are captured by changing the parameters that describe the crystal shape, leading to shape memory effects. Due to the finite growth rate of crystals, the actual crystal shape observed in experiments lags behind the target shape, which would ideally be attained after infinitely long exposure to the nontransient experimental conditions. Using the concept of target shapes, the rela- tion between processing conditions and target shape, which is closely related to experiments and simulations under stationary conditions, is effectively separated from the additional complication of shape memory effects. By way of the dynamic crystallization equations (8b), (10), (12), and (16), respectively, one can extract the target shape from the experimental data on the actual shape, under certain circumstances, as described in Sec. V. Thus, memory effects require combining modeling and experiment to measure and verify the desired constitutive processing-target shape relations.

The intimate relation between crystal shape and thermodynamic driving forces for crystallization was established in Sec. VI. This coupling arises when combining the dynamic crystallization model presented here with a two-phase model, and needs to be included in order to avoid thermodynamic inconsistencies. One finds that the nucleation rate as well as the growth rate have a explicit shape dependence, in addition to being size dependent.

The versatility of the crystallization model presented here is based upon using four structural variables to describe the crystal shape and number density, irrespective of the growth dimensionality. For $d_{\mathrm{G}}$-dimensional growth $\left(d_{\mathrm{G}}=1,2\right.$, 3 ) the number of dynamic variables required for the crystallization model is $d_{\mathrm{G}}+1$. We have shown that an additional $3-d_{\mathrm{G}}$ "static" variables should be included in the model as well (see Secs. III B and III C), e.g., for properly transitioning between two growth regimes of different growth dimensionality, as shown in Sec. III E. The use of multiple structural variables also surfaces in the measurement of the processing-shape relation (Sec. V), as well as in the size and shape dependence of the nucleation rate and growth rate (Sec. VI). A reasonable choice of variables as well as understanding of the structure of their dynamic equations is important to model crystallization successfully under complex processing conditions.

Beyond the illustrative examples discussed in Sec. IV, the morphological description of crystal shape and growth as presented here may also be applied to describe other experimentally observed phenomena. These include distinction between lenticular, truncated lozenge ${ }^{70}$ and hexagonal ${ }^{71}$ shapes.

\section{ACKNOWLEDGMENTS}

Financial support from the Swiss National Science Foundation under Grant No. 81EZ-68591 and from the ERC program of the National Science Foundation under Award No. EEC-9731680 is gratefully acknowledged.

\section{APPENDIX: MINKOWSKI FUNCTIONALS FOR A TRIAXIAL ELLIPSOID}

For a triaxial ellipsoid $\varepsilon$ with main axes $\left(R, \lambda_{1} R, \lambda_{2} R\right)$, the two coefficients $c_{1}(\varepsilon)=\gamma_{1}\left(\lambda_{1}, \lambda_{2}\right)$ and $c_{2}(\varepsilon)=\gamma_{2}\left(\lambda_{1}, \lambda_{2}\right)$ defined by $\boldsymbol{\Psi}_{\mu}(\varepsilon)=c_{\mu}(\varepsilon) R^{3-\mu} / V_{\mathrm{t}}(\mu=1,2)$ are determined. To that end, one can calculate the surface area $\boldsymbol{\Psi}_{1}(\varepsilon)$ as well 
as the surface area of its parallel-body $\boldsymbol{\Psi}_{1}\left(\varepsilon_{\epsilon}\right)$, where the parallel-body $\varepsilon_{\epsilon}$ is defined by

$$
\varepsilon_{\epsilon}:=\{x \mid\|x-y\| \leqslant \epsilon, y \in \varepsilon\}
$$

including the ellipsoid itself as well as all points in the vicinity $\epsilon$ of the ellipsoid. Since it is known from integral geometry $^{13}$ that $\boldsymbol{\Psi}_{2}(\varepsilon)$ can be calculated by

$$
\boldsymbol{\Psi}_{1}\left(\varepsilon_{\epsilon}\right)=\boldsymbol{\Psi}_{1}(\varepsilon)+\epsilon \boldsymbol{\Psi}_{2}(\varepsilon)+O\left(\epsilon^{2}\right),
$$

a straightforward, although lengthy, calculation leads to

$$
\begin{aligned}
& \gamma_{1}\left(\lambda_{1}, \lambda_{2}\right) \\
& =\int_{0}^{\pi} \int_{0}^{2 \pi} \sqrt{\lambda_{1}^{2} \cos ^{2} \theta+\lambda_{2}^{2} \sin ^{2} \theta\left(\sin ^{2} \phi+\lambda_{1}^{2} \cos ^{2} \phi\right)}
\end{aligned}
$$

$\times \sin \theta d \theta d \phi$,

$$
\gamma_{2}\left(\lambda_{1}, \lambda_{2}\right)=\lambda_{1} \lambda_{2} \int_{0}^{\pi} \int_{0}^{2 \pi} \frac{\lambda_{1}^{2} \cos ^{2} \phi+\sin ^{2} \phi+\lambda_{2}^{2} \sin ^{2} \theta+\cos ^{2} \theta\left(\lambda_{1}^{2} \sin ^{2} \phi+\cos ^{2} \phi\right)}{\lambda_{1}^{2} \cos ^{2} \theta+\lambda_{2}^{2} \sin ^{2} \theta\left(\sin ^{2} \phi+\lambda_{1}^{2} \cos ^{2} \phi\right)} \sin \theta d \theta d \phi .
$$

${ }^{1}$ R. R. Lagasse and B. Maxwell, "An experimental study of the kinetics of polymer crystallization during shear flow," Polym. Eng. Sci. 16, 189 (1976).

${ }^{2}$ A. Keller and J. W. H. Kolnaar, "Flow-induced orientation and structure formation," in Processing of Polymers, Material Science and Technology, Vol. 18, edited by H. E. H. Meijer (Wiley-VCH, Weinheim, 1997), pp. 189-268.

${ }^{3}$ G. Eder and H. Janeschitz-Kriegl, "Crystallization," in Processing of Polymers, Material Science and Technology, Vol. 18, edited by H. E. H. Meijer (Wiley-VCH, Weinheim, 1997), pp. 269-342.

${ }^{4}$ J. Petermann, M. Miles, and H. Gleiter, "The crystalline core of the row structures in isotactic polystyrene. I. Nucleation and growth," J. Polym. Sci., Polym. Phys. Ed. 17, 55 (1979).

${ }^{5}$ G. Eder, H. Janeschitz-Kriegl, and S. Liedauer, "Crystallization processes in quiescent and moving polymer melts under heat transfer conditions," Prog. Polym. Sci. 15, 629 (1990).

${ }^{6}$ H. Zuidema, "Flow induced crystallization of polymers," Ph.D. thesis, Eindhoven University of Technology, Eindhoven, The Netherlands, 2000. ${ }^{7}$ H. Zuidema, G. W. M. Peters, and H. E. H. Meijer, "Development and validation of a recoverable strain-based model for flow-induced crystallization of polymers," Macromol. Theory Simul. 10, 447 (2001).

${ }^{8} \mathrm{H}$. Hadwiger, Vorlesungen über Inhalt, Oberfläche und Isoperimetrie (Springer, Heidelberg, 1957).

${ }^{9}$ L. A. Santalò, Integral Geometry and Geometric Probability (AddisonWesley, Reading, 1976).

${ }^{10}$ R. Schneider, Convex Bodies: The Brunn-Minkowski Theory (Cambridge University Press, Cambridge, 1993).

${ }^{11}$ W. Weil, "Stereology: A survey for geometers," in Convexity and Its Applications, edited by P. M. Gruber and J. M. Wills (Birkhäuser, Basel, 1983), pp. 360-412.

${ }^{12}$ K. R. Mecke, "Integral geometry in statistical physics," Int. J. Mod. Phys. B 12, 861 (1998).

${ }^{13}$ K. R. Mecke, "Additivity, convexity, and beyond: Applications of Minkowski functionals in statistical physics," in Statistical Physics and Spatial Statistics: The Art of Analyzing and Modeling Spatial Structures and Pattern Formation, Lecture Notes in Physics Vol. 554, edited by K. R. Mecke and D. Stoyan (Springer, Berlin, 2000), pp. 111-184.

${ }^{14}$ K. R. Mecke, T. Buchert, and H. Wagner, "Robust morphological measures for large-scale structure in the universe," Astron. Astrophys. 288, 697 (1994).

${ }^{15}$ M. Kerscher, K. R. Mecke, P. Schuecker, H. Böhringer, L. Guzzo, C. A. Collins, S. Schindler, S. DeGrandi, and R. Cruddace, "Non-Gaussian morphology on large scales: Minkowski functionals of the REFLEX cluster catalogue," Astron. Astrophys. 377, 1 (2001).

${ }^{16}$ M. Hütter, "Heterogeneity of colloidal particle networks analyzed by means of Minkowski functionals," Phys. Rev. E 68, 031404 (2003).

${ }^{17}$ M. Hütter, "Thermodynamically consistent incorporation of the Schneider rate equations into two-phase models," Phys. Rev. E 64, 011209 (2001).

${ }^{18}$ M. Hütter, "Solidification in closed systems: Cluster size distribution and its driving force," Multiscale Model. Simul. 1, 371 (2003).

${ }^{19}$ A. N. Kolmogoroff, "On the statistical theory of the crystallization of metals," Bull. Acad. Sci. USSR 1, 355 (1937).

${ }^{20}$ W. A. Johnson and R. F. Mehl, "Reaction kinetics in processes of nucleation and growth," Trans. Am. Inst. Min., Metall. Pet. Eng. 135, 416 (1939).

${ }^{21}$ M. Avrami, "Kinetics of phase change. I. General theory," J. Chem. Phys. 7, 1103 (1939).

${ }^{22}$ M. Avrami, "Kinetics of phase change. II. Transformation-time relations for random distribution of nuclei," J. Chem. Phys. 8, 212 (1940).

${ }^{23} \mathrm{M}$. Avrami, "Kinetics of phase change. III. Granulation, phase change, and microstructure," J. Chem. Phys. 9, 177 (1941).

${ }^{24}$ W. Schneider, A. Köppel, and J. Berger, "Non-isothermal crystallization of polymers," Int. Polym. Process. 2, 151 (1988).

${ }^{25}$ M. Hütter, "GENERIC treatment of compressible two-phase flow: Convection mechanism of scalar morphological variables," J. Non-Equil. Thermodyn. 27, 349 (2002).

${ }^{26}$ M. Ishii, Thermo-Fluid Dynamic Theory of Two-Phase Flow (Eyrolles, Paris, 1975).

${ }^{27}$ D. A. Drew, "Mathematical modeling of two-phase flow," Annu. Rev. Fluid Mech. 15, 261 (1983).

${ }^{28}$ C. Morel, N. Goreaud, and J.-M. Delhaye, "The local volumetric interfacial area transport equation: Derivation and physical significance," Int. J. Multiphase Flow 25, 1099 (1999).

${ }^{29}$ N. J. Wagner, H. C. Öttinger, and B. J. Edwards, "Generalized Doi-Ohta model for multiphase flow developed via GENERIC," AIChE J. 45, 1169 (1999).

${ }^{30} \mathrm{D}$. Lhuillier, "A mean-field description of two-phase flows with phase changes," Int. J. Multiphase Flow 29, 511 (2003).

${ }^{31}$ M. Hütter, "Solidification in closed systems: Cluster size distribution and its driving force," Multiscale Model. Simul. 1, 371 (2003).

${ }^{32}$ R. I. Nigmatulin, Dynamics of Multiphase Media (Hemisphere, New York, 1990).

${ }^{33}$ A. Toda, T. Arita, C. Tomita, and M. Hikosaka, "Temperature-modulated DSC applied to the transformation kinetics of polymer crystallization," Polym. J. (Tokyo, Jpn.) 31, 790 (1999).

${ }^{34}$ M. Stadlbauer, G. Eder, and H. Janeschitz-Kriegl, "Crystallization kinetics of two aliphatic polyketones," Polymer 42, 3809 (2001).

${ }^{35}$ J. J. Hoyt and M. Asta, "Atomistic computation of liquid diffusivity, solid-liquid interfacial free energy, and kinetic coefficient in Au and Ag," Phys. Rev. B 65, 214106 (2002).

${ }^{36}$ N. Waheed, M. S. Lavine, and G. C. Rutledge, "Molecular simulation of crystal growth in n-eicosane," J. Chem. Phys. 116, 2301 (2002).

${ }^{37}$ M. S. Lavine, N. Waheed, and G. C. Rutledge, "Molecular dynamics simulation of orientation and crystallization of polyethylene during uniaxial extension," Polymer 44, 1771 (2003).

${ }^{38}$ M. J. Ko, N. Waheed, M. S. Lavine, and G. C. Rutledge, "Characterization of polyethylene crystallization from an oriented melt by molecular dynamics simulation," J. Chem. Phys. 121, 2823 (2004).

${ }^{39}$ J. A. Kornfield, G. Kumaraswamy, and A. M. Issaian, "Recent advances in understanding flow effects on polymer crystallization," Ind. Eng. Chem. Res. 41, 6383 (2002)

${ }^{40}$ M. Hikosaka, K. Amano, S. Rastogi, and A. Keller, "Lamellar thickening 
growth of an extended chain single crystal of polyethylene. 1. Pointers to a new crystallization mechanism of polymers," Macromolecules 30, 2067 (1997).

${ }^{41}$ M. Hikosaka, K. Amano, S. Rastogi, and A. Keller, "Lamellar thickening growth of an extended chain single crystal of polyethylene (II): Delta T dependence of lamellar thickening growth rate and comparison with lamellar thickening," J. Mater. Sci. 35, 5157 (2000).

${ }^{42}$ E. W. Fischer and G. F. Schmidt, "Über Langperioden bei verstrecktem Polyäthylen," Angew. Chem. 74, 551 (1962).

${ }^{43}$ J. Rault and E. Robelin-Souffaché, "Long-periods in slow-cooled linear and branched polyethylene: Part II," J. Polym. Sci., Part B: Polym. Phys. 27, 1349 (1989).

${ }^{44}$ J. van Meerveld, G. W. M. Peters, and M. Hütter, "Towards a rheological classification of flow induced crystallization experiments of polymer melts," Rheol. Acta (in press).

${ }^{45}$ I. Lieberwirth, J. Loos, J. Petermann, and A. Keller, "Observation of shish crystal growth into nondeformed melts," J. Polym. Sci., Part B: Polym. Phys. 38, 1183 (2000)

${ }^{46}$ J. M. Schultz, Polymer Crystallization. The Development of Crystalline Order in Thermoplastic Polymers (Oxford University Press, Oxford, 2001).

${ }^{47}$ J. M. Schultz and J. Petermann, "Transmission electron microscope observations of fibrillar-to-lamellar transformations in melt-drawn polymers-I. Isotactic polypropylene," Colloid Polym. Sci. 262, 294 (1984).

${ }^{48}$ K. G. Lee and J. M. Schultz, "The development of structure and mechanical properties in poly(ethylene terephtalate) fibers during heat-treatment under stress," Polymer 34, 4455 (1993).

${ }^{49}$ P. N. Pusey, "Introduction to scattering experiments," in Neutrons, X-rays and Light: Scattering Methods Applied to Soft Condensed Matter, edited by P. Lindner and Th. Zemp (North-Holland, Amsterdam, 2002), pp. 3-21.

${ }^{50}$ G. Porod, "Die Röntgenkleinwinkelstreuung von dichtgepackten kolloiden Systemen (X-ray small angle diffraction of closely packed colloidal systems)," Kolloid-Z. 124, 83 (1951).

${ }^{51}$ G. Porod, "Die Röntgenkleinwinkelstreuung von dichtgepackten kolloiden Systemen (X-ray small angle diffraction of closely packed colloidal systems)," Kolloid-Z. 125, 51 (1951).

${ }^{52}$ S. Ciccariello, J. M. Schneider, B. Schönfeld, and G. Kostorz, "Generalization of Porod's law of small-angle scattering to anisotropic samples," Europhys. Lett. 50, 601 (2000).

${ }^{53}$ J. M. Schneider, S. Ciccariello, B. Schönfeld, and G. Kostorz, "The asymptotic leading term of anisotropic small-angle scattering intensities. II. Non-convex particles," Acta Crystallogr., Sect. A: Found. Crystallogr. 58, 221 (2002).

${ }^{54} \mathrm{O}$. Glatter, "The inverse scattering problem in small-angle scattering," in Neutrons, X-rays and Light: Scattering Methods Applied to Soft Condensed Matter, edited by P. Lindner and Th. Zemp (North-Holland, Amsterdam, 2002), pp. 73-102.
${ }^{55}$ J. S. Pedersen, "Modelling of small-angle scattering data from colloids and polymer systems," in Neutrons, X-rays and Light: Scattering Methods Applied to Soft Condensed Matter, edited by P. Lindner and Th. Zemp (North-Holland, Amsterdam, 2002), pp. 391-420.

${ }^{56}$ Lord Rayleigh, "The incidence of light upon a transparent sphere of dimensions comparable with the wave-length," Proc. R. Soc. London, Ser. A 84, 25 (1911).

${ }^{57} \mathrm{~A}$. Guinier, "La diffraction des rayons $\mathrm{X}$ aux tres petits angles: Application à l'étude de phenomenes ultramicroscopiques," Ann. Phys. (Paris) 12, 161 (1939).

${ }^{58}$ P. Mittelbach and G. Porod, "X-ray small-angle scattering of dilute colloidal systems. VII. Computations for scattering curves of triaxial ellipsoids," Acta Phys. Austriaca 15, 122 (1962).

${ }^{59}$ P. Mittelbach and G. Porod, "Zur Röntgenkleinwinkelstreuung verdünnter kolloider Systeme," Acta Phys. Austriaca 14, 185 (1961).

${ }^{60}$ G. Fournet, "Scattering functions for geometrical forms," Bull. Soc. Fr. Mineral. Cristallogr. 74, 39 (1951).

${ }^{61}$ B. Wunderlich, Macromolecular Physics, Vol. 2: Crystal Nucleation, Growth Annealing (Academic, New York, 1976).

${ }^{62} \mathrm{~K}$. Ravindranath and J. P. Jog, "Polymer crystallization kineticspoly(ethylene-terephthalate) and poly(phenylene sulfide)," J. Appl. Polym. Sci. 49, 1395 (1993).

${ }^{63}$ M. Hütter, "Crystallization under external pressure," J. Non-Newtonian Fluid Mech. 120, 55 (2004).

${ }^{64}$ R. J. J. Jongschaap, "Microscopic modeling of the flow properties of polymers," Rep. Prog. Phys. 53, 1 (1990).

${ }^{65}$ R. J. J. Jongschaap, "Towards a unified formulation of microrheological models," in Rheological Modelling: Thermodynamical and Statistical Approaches, Lecture Notes in Physics, Vol. 381, edited by J. Casas-Vázquez and D. Jou (Springer, Berlin, 1991), pp. 215-247.

${ }^{66}$ R. J. J. Jongschaap, K. de Haas, and C. Damen, "A generic matrix representation of configuration tensor rheological models," J. Rheol. 38, 769 (1994).

${ }^{67}$ M. Grmela and H. C. Öttinger, "Dynamics and thermodynamics of complex fluids. I. Development of a general formalism," Phys. Rev. E 56, 6620 (1997).

${ }^{68}$ H. C. Öttinger and M. Grmela, "Dynamics and thermodynamics of complex fluids. II. Illustrations of a general formalism," Phys. Rev. E 56, 6633 (1997).

${ }^{69}$ K. Nakamura, K. Katayama, and T. Amoto, "Some aspects of nonisothermal crystallization of polymers. II. Consideration of the isokinetic condition,” J. Appl. Polym. Sci. 17, 1031 (1973).

${ }^{70}$ A. Toda and A. Keller, "Growth of polyethylene single crystals from the melt: morphology," Colloid Polym. Sci. 271, 328 (1993).

${ }^{71}$ Y. Tanzawa, "Growth-rate and morphology of isotactic polystyrene crystals in solution at high supercoolings," Polymer 33, 2659 (1992). 